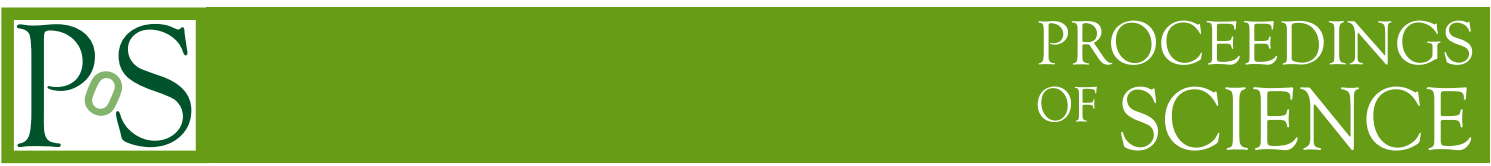

\title{
Measurement of radiative widths at COMPASS
}

\author{
Markus Krämer*ifor the COMPASS Collaboration \\ Technische Universität München \\ E-mail: mkraemer@tum.de
}

\begin{abstract}
COMPASS is a multipurpose fixed-target experiment at the CERN SPS, which addresses a wide variety of physic topics, in particular the structure and spectroscopy of hadrons. Diffractive dissociation of pions on nuclear targets allows for clean access to the light meson spectrum.

In addition meson production can be studied in pion-photon reactions via the Primakoff effect, where high-energetic pions react with the quasi-real photon field surrounding the target nuclei. At low pion-photon center-of-mass energies, these reactions are governed by chiral dynamics and contain information relevant for chiral perturbation theory. At higher energies, resonances are produced and their radiative coupling is investigated.

During a short run using a $190 \mathrm{GeV} \pi^{-}$beam and a lead target in the year 2004, 3 million exclusive $\pi^{-} \pi^{-} \pi^{+}$events in the region of small squared four-momentum transfer, i.e. $t^{\prime}<0.01 \mathrm{GeV}^{2} / c^{2}$, have been recorded. At very low $t^{\prime}<0.001 \mathrm{GeV}^{2} / c^{2}$, the contribution from electromagnetic interactions becomes visible. A partial-wave analysis of the data was performed, clean signals for the $a_{2}(1320)$ and the $\pi_{2}(1670)$ were observed. The radiative couplings of the $a_{2}(1320)$ and, for the first time, that of the $\pi_{2}(1670)$ were measured by isolating the electromagnetic contribution to the corresponding waves.
\end{abstract}

XV International Conference on Hadron Spectroscopy-Hadron 2013

4-8 November 2013

Nara, Japan

\footnotetext{
* Speaker.

$\dagger$ The author acknowledges financial support by the organisators of the conference, the German Bundesministerium für Bildung und Forschung (BMBF), by the Maier-Leibnitz-Laboratorium der LMU und TU München, and by the DFG cluster of excellence 'Origin and Structure of the Universe'
} 


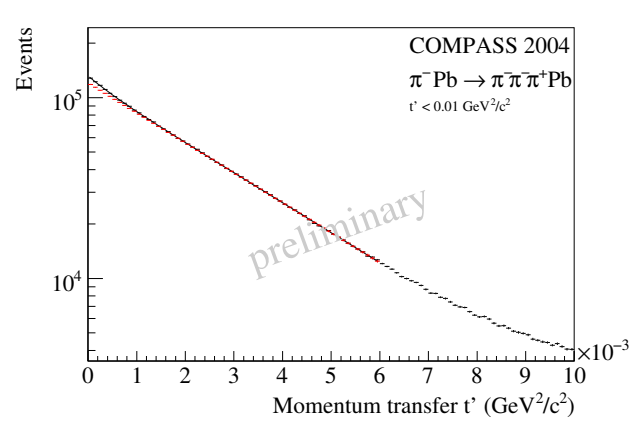

(a)

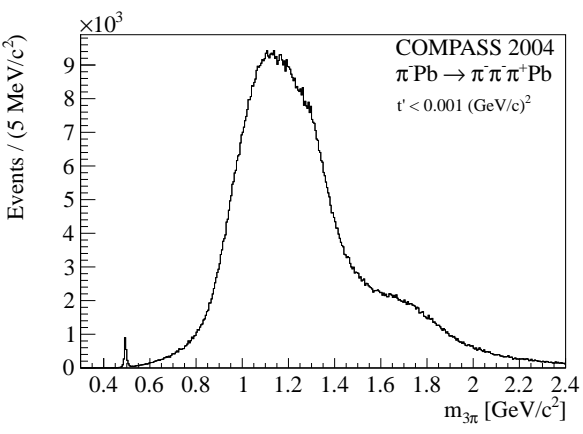

(b)

Figure 1: COMPASS 2004: (a) Momentum transfer distribution of $\pi^{-} \mathrm{Pb} \rightarrow \pi^{-} \pi^{-} \pi^{+} \mathrm{Pb}$. The red points indicate the determined shape of diffractive production. (b) Invariant mass spectrum of $\pi^{-} \pi^{-} \pi^{+}$final state for $t^{\prime}<0.001 \mathrm{GeV}^{2} / c^{2}$. The sharp peak at $m_{3 \pi}=0.493 \mathrm{GeV} / c^{2}$ originates from the decay of beam kaons.

\section{Introduction}

An important tool to study the electric and magnetic current distribution within mesons and therefore their internal structure is to measure their radiative decay. Here the measurement of the radiative decays $a_{2}(1320) \rightarrow \pi \gamma$, a magnetic quadrupole transition, and $\pi_{2}(1670) \rightarrow \pi \gamma$, an electric quadrupole transition, at COMPASS will be described. There are several measurements (see [1], [2] and [3]) and predictions using various models (see [4], [5] and [6]) for the radiative width $\Gamma_{0}\left(a_{2}(1320) \rightarrow \pi \gamma\right)$, where the COMPASS measurement can contribute. There are no measurements of $\Gamma_{0}\left(\pi_{2}(1670) \rightarrow \pi \gamma\right)$ published yet, thus the here presented measurement will be the first of this quantity. It can be compared to model predictions, which could be found in [7].

The direct measurement of radiative decays of a resonance $X$ to a $\pi \gamma$ final state is due to the typically low cross-section and numerous background processes difficult to accomplish. In COMPASS, the measurement is accomplished by studying Primakoff production of the resonance $X$ in $\pi \gamma$ scattering and its decay to the $\pi^{-} \pi^{-} \pi^{+}$final state embeded in $\pi^{-}$-Lead scattering. During the short period in 2004, in which the data analyzed here were recorded, a $190 \mathrm{GeV} \pi^{-}$beam was used to record approximately 3 million exclusive $\pi^{-} \pi^{-} \pi^{+}$events in the region of small squared four-momentum transfer, i.e. $t^{\prime}<0.01 \mathrm{GeV}^{2} / c^{2}$. Figure 1 shows the distribution of the transferred momentum $t^{\prime}$ and the invariant mass $m_{3 \pi}$ of the three pion final state.

\section{Partial-Wave Analysis}

The intensity of the resonances was extracted performing a partial-wave analysis. An isobaric model, assuming intermediate two-particle decays (see figure 2), is used and acceptance correction are applied. Partial waves are defined by $J^{P C} M^{\varepsilon}$ \{isobar\} $[L] \pi$ with $J^{P C} M^{\varepsilon}$ defining the quantum numbers of the resonance $X$ and $\{$ isobar $\}[L] \pi$ describing the decay mode.

For very low $t^{\prime}<0.001 \mathrm{GeV}^{2} / c^{2}$ Primakoff production is dominant for waves with spin projection $M=1$. In this region of $t^{\prime}$ a so-called mass-independent partial-wave analysis is done in $40 \mathrm{MeV} / c^{2}$ bins of the invariant mass of the outgoing three-pion system. Figure 3 shows the extracted intensity of the $2^{++} 1^{+} \rho[D] \pi$ wave, which is relevant for the measurement of the radiative 


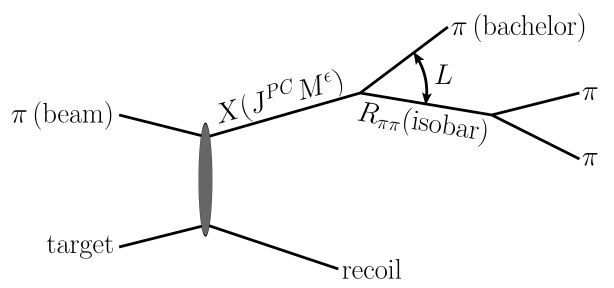

Figure 2: Sketch of an isobaric three-pion decay.

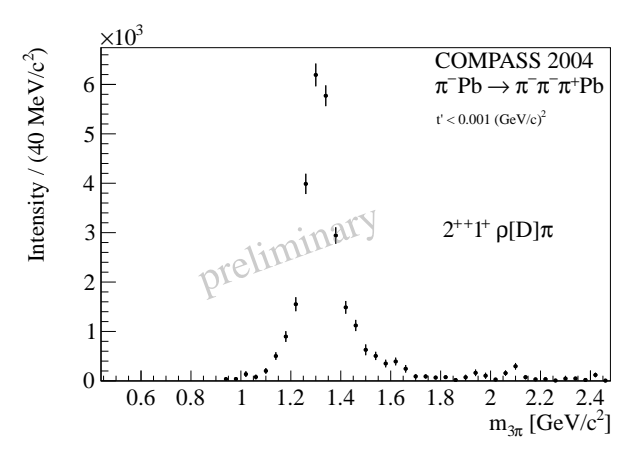

(a)

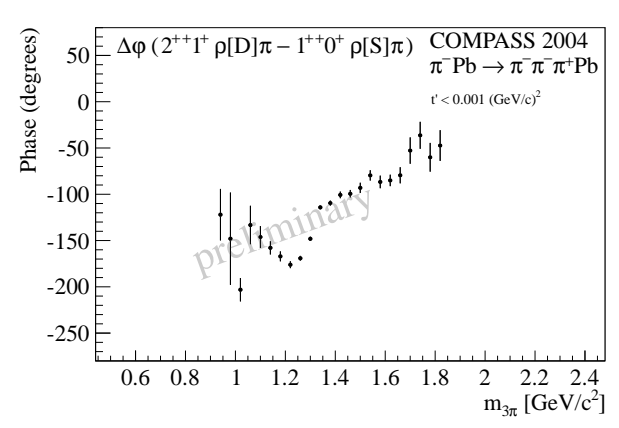

(b)

Figure 3: (a) Intensity in bins of $m_{3 \pi}$ of the $2^{++} 1^{+} \rho[\mathrm{D}] \pi$ wave, (b) Relative phase $\Delta \phi\left(2^{++} 1^{+} \rho[\mathrm{D}] \pi-\right.$ $\left.1^{++} 0^{+} \rho[\mathrm{S}] \pi\right)$ in bins of $m_{3 \pi}$.

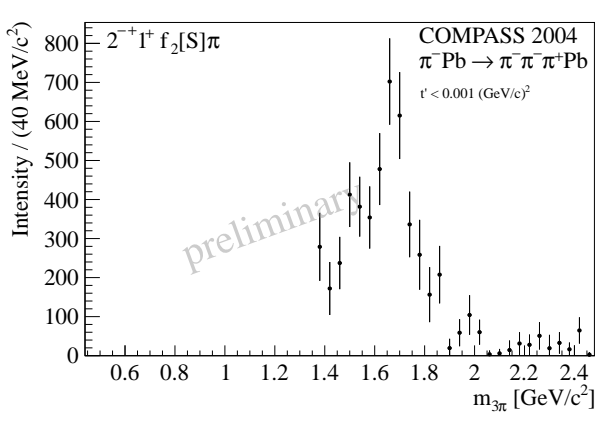

(a)

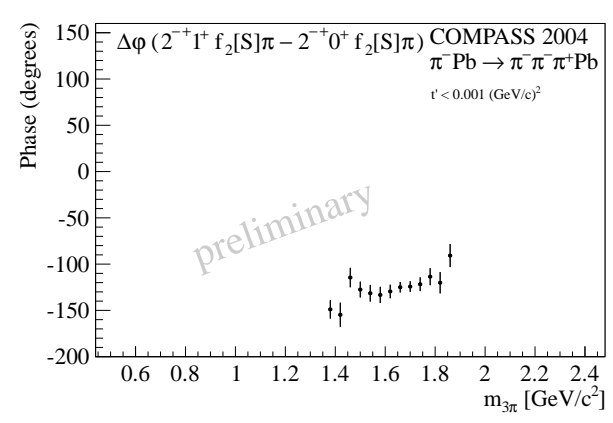

(b)

Figure 4: (a) Intensity in bins of $m_{3 \pi}$ of the $2^{-+} 1^{+} \mathrm{f}_{2}[\mathrm{~S}] \pi$ wave, (b) Relative phase $\Delta \phi\left(2^{-+} 1^{+} \mathrm{f}_{2}[\mathrm{~S}] \pi-\right.$ $\left.2^{-+} 0^{+} f_{2}[S] \pi\right)$ in bins of $m_{3 \pi}$.

width of the $a_{2}(1320)$, and its relative phase to the $1^{++} 0^{+} \rho[S] \pi$ wave, which is dominated by the diffractively produced $a_{1}(1260)$. The $a_{2}(1320)$ can be observed in intensity and phase motion. Figure 4 shows the intensity of the $2^{-+} 1^{+} f_{2}[S] \pi$ wave, which contains the mainly Primakoff produced $\pi_{2}(1670)$ and its phase difference relative to the $2^{-+} 0^{+} f_{2}[S] \pi$ wave, which is mainly diffractive produced $\pi_{2}(1670)$.

In order to account for the diffractive produced background a partial wave analysis in bins of $t^{\prime}$ around the 3 pion mass of the resonance $X$ is done (see Figure 5 and 6). A fit to the intensity distribution determines the admixture of Primakoff and diffractive production. 


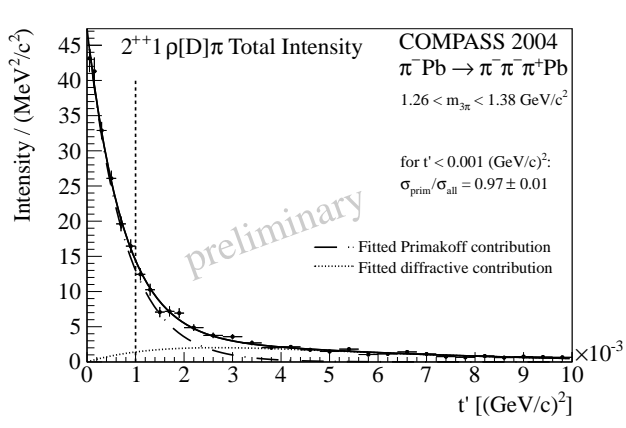

(a)

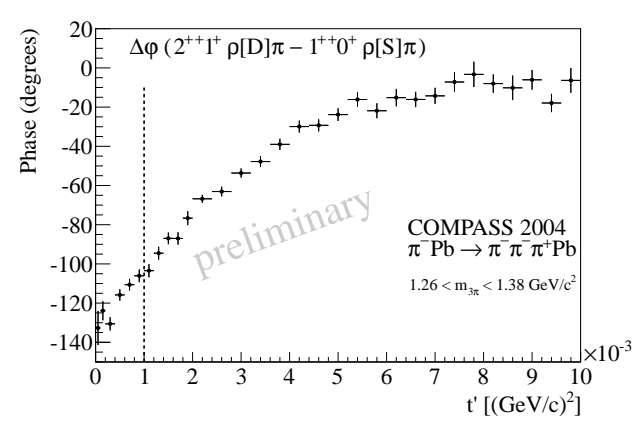

(b)

Figure 5: (a) Intensity in bins of $t^{\prime}$ of the $2^{++} 1^{+} \rho[\mathrm{D}] \pi$ wave in mass-range $1.26 \mathrm{GeV} / c^{2}<m_{3 \pi}<$ $1.38 \mathrm{GeV} / c^{2}$ (b) Relative phase $\Delta \phi\left(2^{++} 1^{+} \rho[\mathrm{D}] \pi-1^{++} 0^{+} \rho[\mathrm{S}] \pi\right.$ in bins of $t^{\prime}$ in the same $m_{3 \pi}$ region.

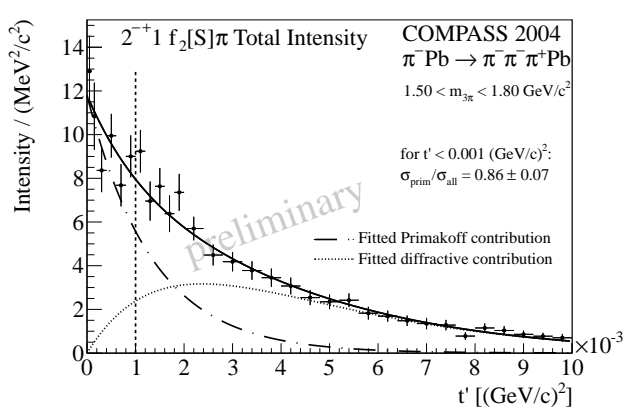

(a)

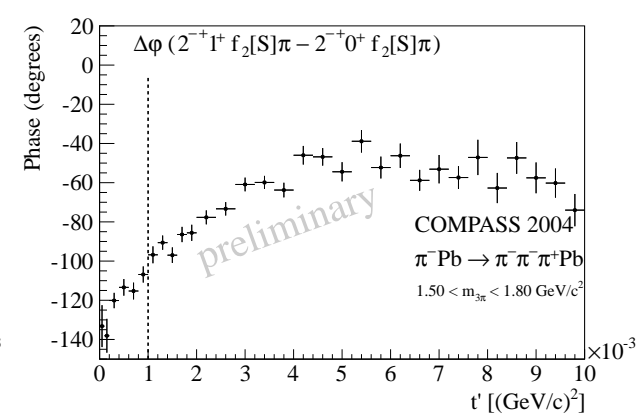

(b)

Figure 6: (a) Intensity in bins of $t^{\prime}$ of the $2^{++} 1^{+} \rho[\mathrm{D}] \pi$ wave in mass-range $1.50 \mathrm{GeV} / c^{2}<m_{3 \pi}<$ $1.80 \mathrm{GeV} / c^{2}$ (b) Relative phase $\Delta \phi\left(2^{++} 1^{+} \rho[\mathrm{D}] \pi-1^{++} 0^{+} \rho[\mathrm{S}] \pi\right.$ in bins of $t^{\prime}$ in the same $m_{3 \pi}$ region.

\section{Primakoff Production of a broad Resonance}

The differential cross section for Primakoff production of a broad resonance $X$ is given by

$$
\frac{\mathrm{d} \sigma}{\mathrm{d} m \mathrm{~d} t^{\prime}}=16 \alpha Z^{2}\left(\frac{m}{m^{2}-m_{\pi}^{2}}\right)^{3} \frac{m_{0}^{2} \Gamma_{\pi \gamma}(m) \Gamma_{\text {final }}(m)}{\left(m^{2}-m_{0}^{2}\right)^{2}+m_{0}^{2} \Gamma_{\text {total }}(m)^{2}} \frac{t^{\prime}}{\left(t^{\prime}+t_{\text {min }}\right)^{2}}\left|F\left(t^{\prime}\right)\right|^{2} .
$$

$\Gamma_{\text {total }}(m)$ denotes the the total mass-dependent width of the resonance $X . \Gamma_{\text {final }}(m)$ is the massdependent width of resonance $X$ decaying to the investigated final state. Here, $\Gamma_{\pi \gamma}(m)=f_{\pi \gamma}^{d y n}(m)$. $\Gamma_{0}(X \rightarrow \pi \gamma)$ is the mass-dependent radiative width, with the factor $f_{\pi \gamma}^{d y n}(m)$ accounting for the mass-dependence.

Thus the total cross-section in a certain mass and $t^{\prime}$ range is on the one hand given by the product of a normalization integral $C_{X}$ and the radiative width $\Gamma_{0}(X \rightarrow \pi \gamma)$ of the resonance.

$$
\sigma_{\text {Primakoff }}=\int_{m_{1}}^{m_{2}} \int_{t_{1}^{\prime}=0}^{t_{2}^{\prime}} \frac{\mathrm{d} \sigma}{\mathrm{d} m \mathrm{~d} t^{\prime}} \mathrm{d} t^{\prime} \mathrm{d} m=\Gamma_{0}(X \rightarrow \pi \gamma) \cdot C_{X},
$$

and on the other hand it can be determined experimentally as 


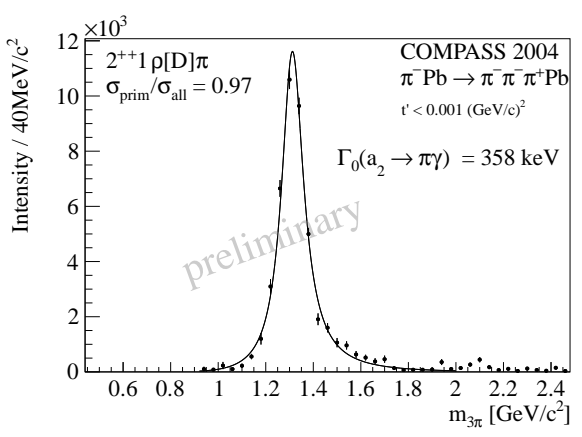

(a)

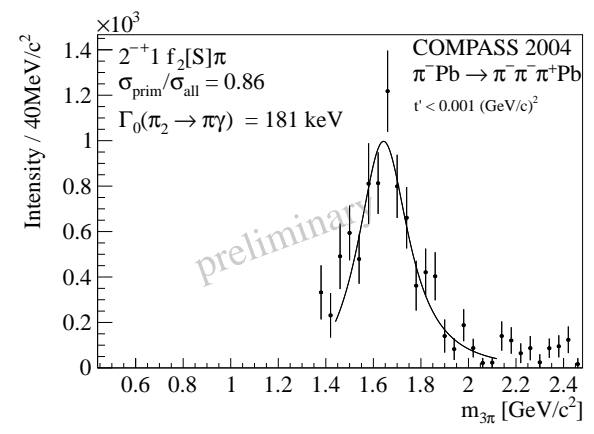

(b)

Figure 7: (a) Total waves intensities $J^{P C} M=2^{++} 1 \rho[\mathrm{D}] \pi$ decays with Breit-Wigner fit (b) Total waves intensities $J^{P C} M=2^{-+} 1 \mathrm{f}_{2}[\mathrm{~S}] \pi$ with a Breit-Wigner fit.

$$
\sigma_{\text {Primakoff }}=\frac{N_{x} / \varepsilon}{\mathscr{L} \cdot C G^{2} \cdot B R\left(X \rightarrow \pi^{-} \pi^{-} \pi^{+}\right) \cdot \varepsilon_{\text {resol }}} .
$$

Thereby the integrated luminosity $\mathscr{L}$ is measured analyzing decays of beam kaons. The branching fraction of the resonance $X$ decaying into the three-pion final state $B R\left(X \rightarrow \pi^{-} \pi^{-} \pi^{+}\right)$is taken from the PDG [8], $C G$ is the respective Clebsch-Gordan coefficient. $\varepsilon_{\text {resol }}$ accounts for effects of the experimental resolution. The quantity $N_{x} / \varepsilon$ is determined by fitting Breit-Wigners to the acceptancecorrected intensity obtained by the partial-wave analysis. The radiative width of the $a_{2}(1320)$ is extracted by fitting the mass-dependence of the $J^{P C} M=2^{++} 1 \rho[\mathrm{D}] \pi$ total waves intensities and that of the $\pi_{2}(1670)$ by fitting the mass-dependence of the $J^{P C} M=2^{-+} 1 \mathrm{f}_{2}[\mathrm{~S}] \pi$ total waves intensities (Figure 7). For the radiative width, one gets $\Gamma_{0}\left(a_{2}(1320) \rightarrow \pi \gamma\right)=(358 \pm 6 \pm 42) \mathrm{keV} / c^{2}$ and $\Gamma_{0}\left(\pi_{2}(1670) \rightarrow \pi \gamma\right)=(181 \pm 11 \pm 27) \mathrm{keV} / c^{2} \cdot 0.56 / B R_{f_{2}} \pi$. In case of the $\pi_{2}(1670)$, the factor $0.56 / B R_{f_{2}} \pi$ accounts for the uncertainty of the branching ratio $B R_{f_{2}} \pi$, which was given as 0.56 by PDG in the current analysis. A paper, which will describe this analysis in greater details, is currently in preparation.

\section{References}

[1] E. May et al., Photoproduction of $\eta \pi^{ \pm}$resonances, Phys. Rev. D 16 (7) 1983-1985.

[2] S. Cihangir et al., Radiative width of the A2(1310), Phys. Lett. B 117 (1-2) 119-122.

[3] V.V. Molchanov et al., Radiative decay width of the a2(1320)- meson, Phys. Lett. B 521 (3-4) 171-180.

[4] M. Singer et al., Photoproduction of $\eta \pi^{ \pm}$resonances, Phys. Rev. D 16 (7) 1983-1985.

[5] I. G. Aznauryan and K. A. Ogamesyan, Sov. J. Nucl. Phys. 47 (1097).

[6] S. Ishida, K. Yamada and M. Oda, Radiative decays of light-quark S-and P-wave mesons in the covariant oscillator quark model, Phys. Rev. D 40 (5) 1497-1512.

[7] T. Maeda, K. Yamada, M. Oda and S. Ishida, Radiative $\pi^{ \pm} \gamma$ transitions of excited light-quark mesons in the covariant oscillator quark model, arXiv:1310.7507 [hep-ph].

[8] K. Nakamura et al. (Particle Data Group), Review of Particle Physics, J. Phys. G: Nucl. Part. Phys. 37 075021. 